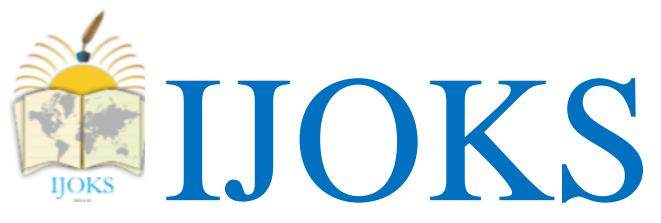

International Journal of Kurdish Studies

(ISSN:2149-2751)

4 (1), pp. $28-43$

http://www.ijoks.com

\title{
The Classification of vowels and consonants in Syllable structure
}

\section{Dilshad M. GHARIB ${ }^{1}$ \& Mhemed MEHWI}

Received: Oct 08, 2017 Reviewed: Oct 30, 2017 Accepted: Nov 05, 2017

\begin{abstract}
This study entitled ( The Classification of vowels and consonants in Syllable structures). It is a part of researchers PhD Dissertation entitled ( stress and accent in karkuk sub-dialect). Here, the researcher attempts to clarity and place the vowels and consonants in the structure and positions of those syllable by using tree diagram model the constituent model of classifying syllable structure for this scientific research. In the first model (tree diagram) the vowel (peak) or (nucleus) placed in the middle or center of the syllable under the rhymes and the onset and coda usually placed at the beginning or the end of the syllable.

This study consists of an introduction and two parts. The results and conclusions, with the abstract in both English and Arabic languages are presented at the end of the study. In part one is dedicated to the classification of vowels and consonants in Syllable structure.In the second part, the structure of vowels are clarified however, some basic tenets about consonant clusters at the beginning or the end of the word is mentioned.The samples and data had proved that it is not possible to have a zero onset and it is also impossible to have more than two consonant clusters initially. At the same time, in final position, it is possible to have zero coda, but it is impossible to have more than two allowed consonant clusters finally. The peak or nucleus of the Syllable can be either strong or weak Syllable.
\end{abstract}

Keywords: Syllable Structure, Sonority, Consonant, Vowel Length, Stress, CV- Model

\section{Recommended citation:}

Gharib D.M. \& Mehwi, M.A. (2018). The Classification of vowels and consonants in Syllable structure. International Journal of Kurdish Studies 4 (1), 1 - 27, DOI:

\footnotetext{
${ }^{1}$ Assitant, Department of Kurdish Language, College of Language speciality: PH.D Language Sulaimani University, Sulaimani, Kurdistan Region - Iraq.

2. Prof. Dr, Department of Kurdish Language, College of Sulaimani University, Sulaimani, Kurdistan Region Iraq.
} 


\section{קֶِيش}

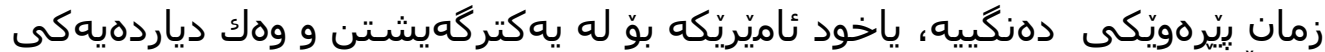

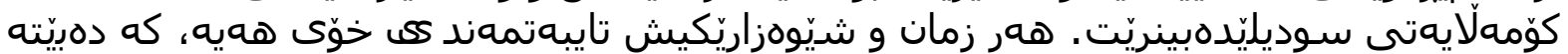

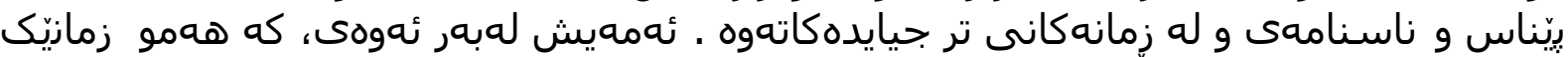

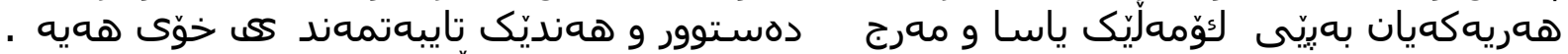

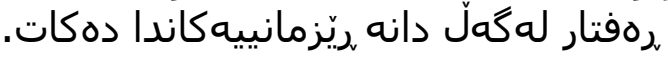

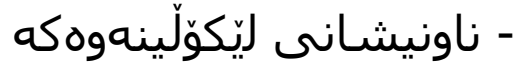

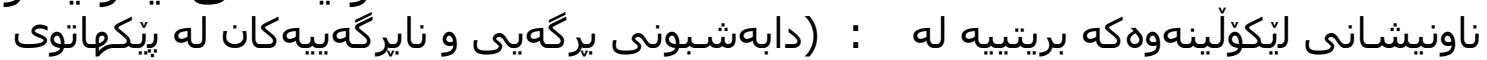

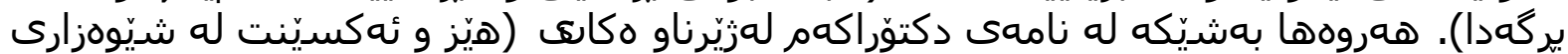

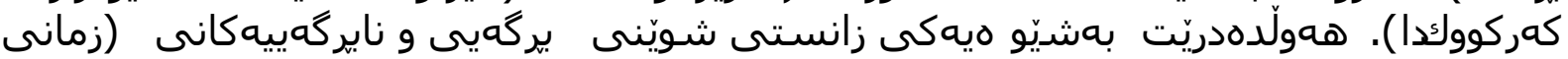

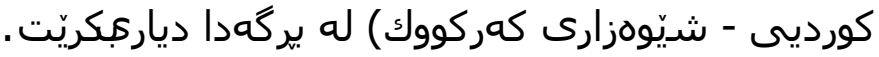

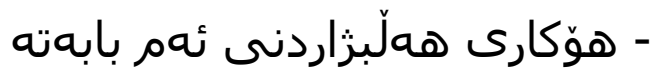

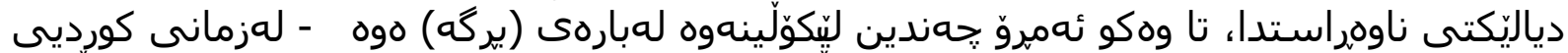

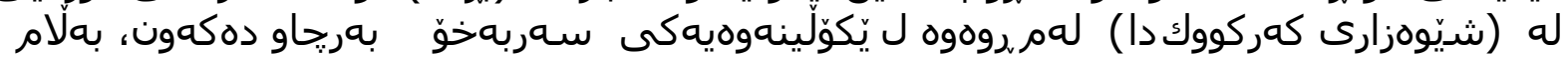

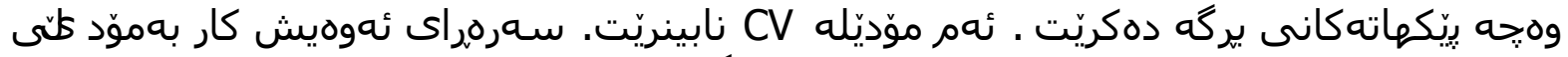

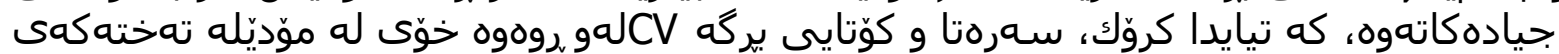

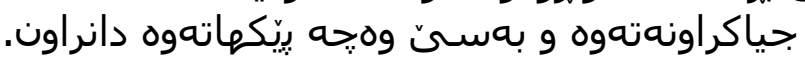

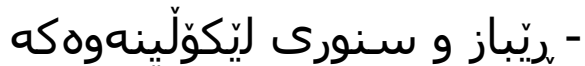

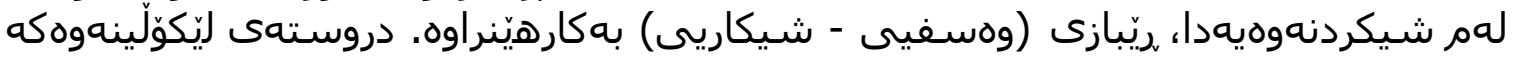

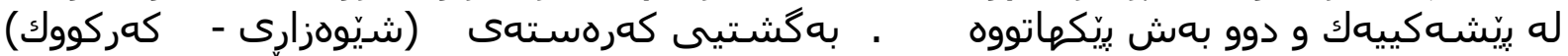

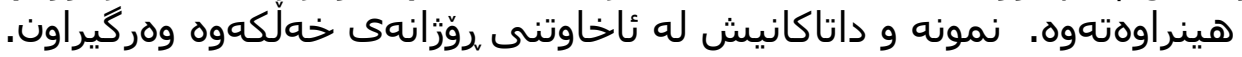

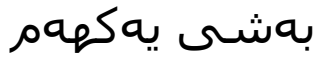

فوَّيّمهكانى زمانى كورديى (شديّوهزارى كهركووك)

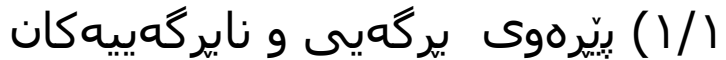

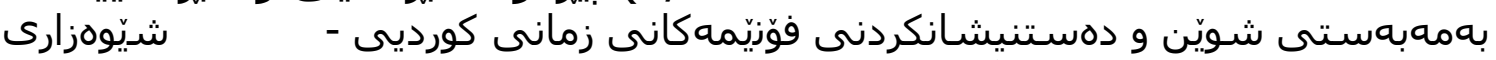

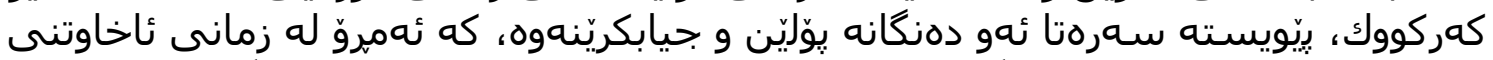

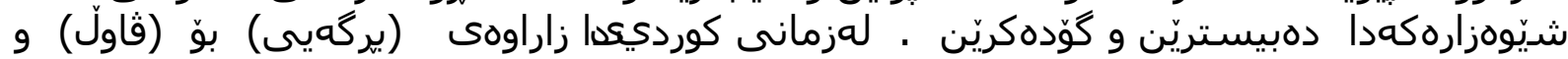

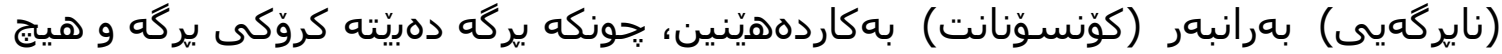

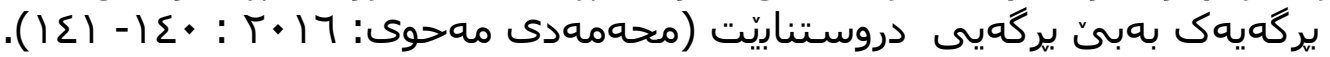

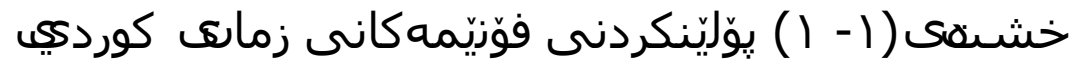

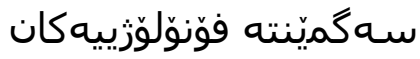

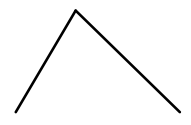

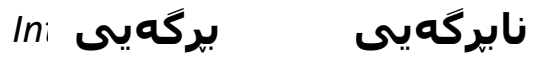




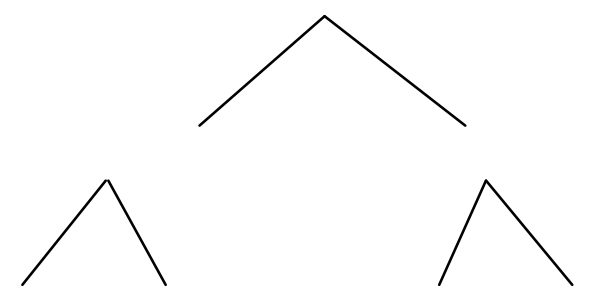

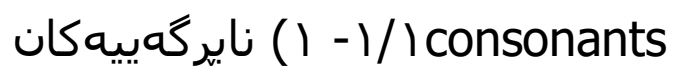

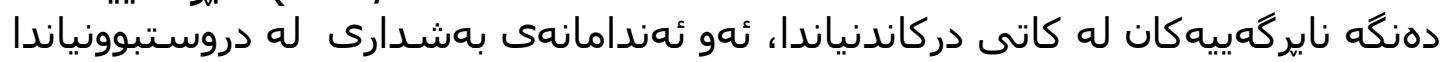

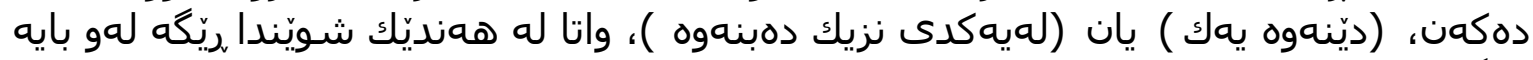

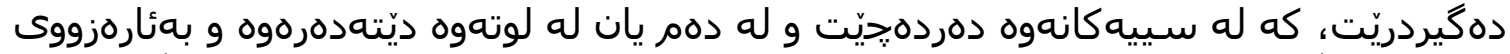

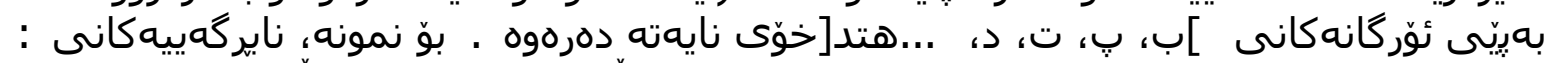

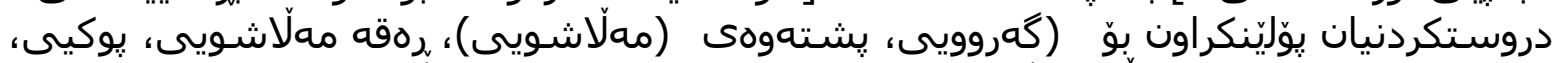

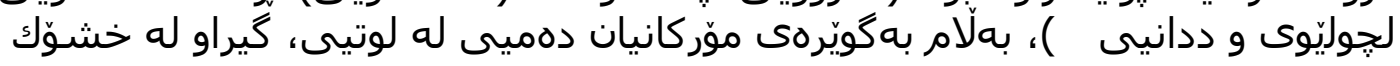

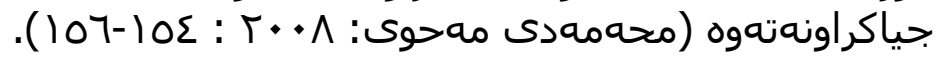

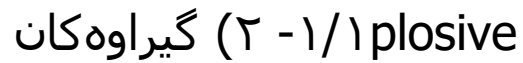

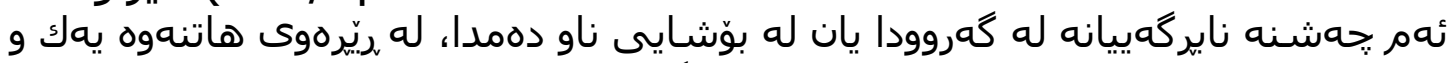

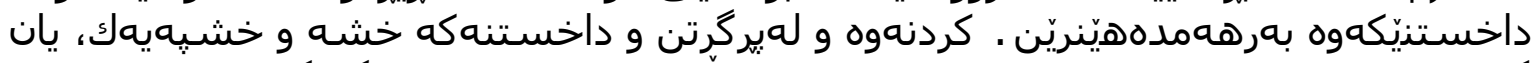

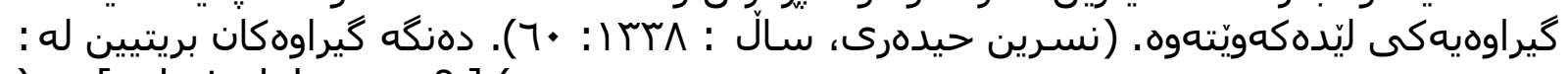
) $[p, b, t, d, k, g, q, ?] .($

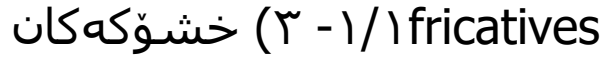

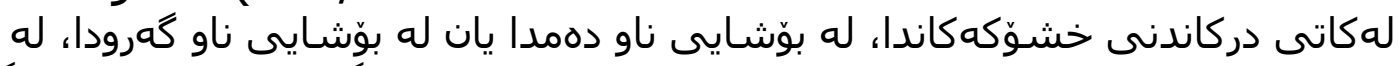

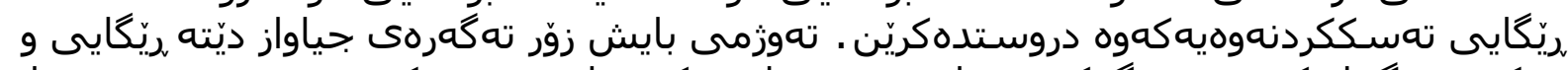

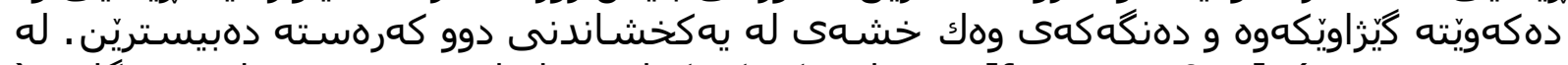

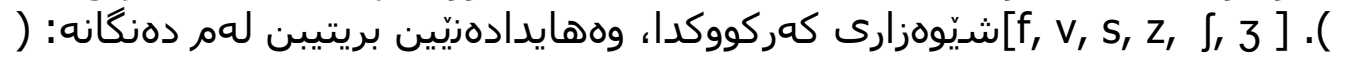

\section{( ) / laffricate}

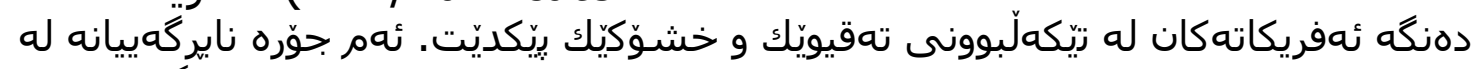

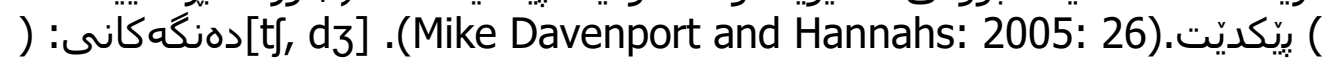

\section{( / / nasals}

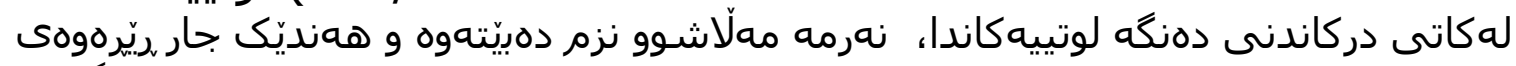

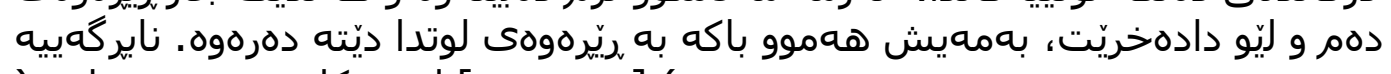

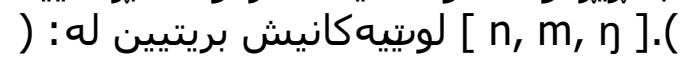

\section{( / / lateral sounds}

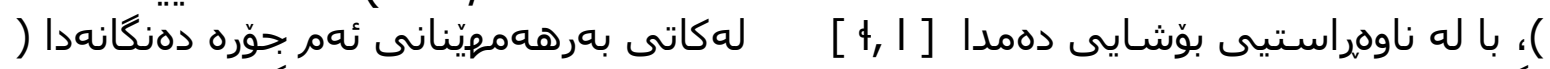

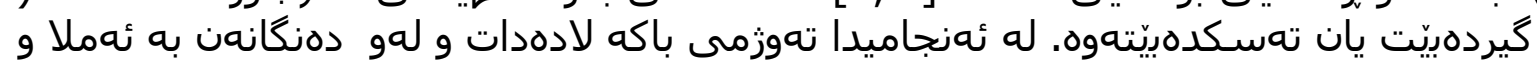

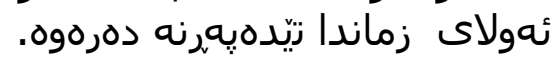


(V - l/ l vibrants sounds

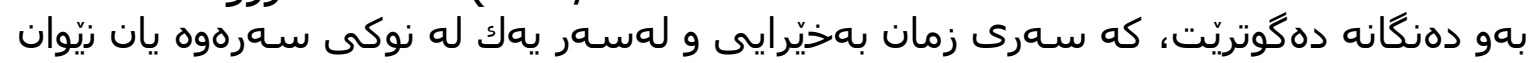

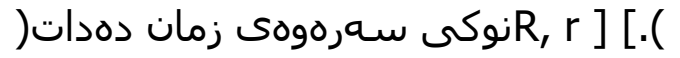

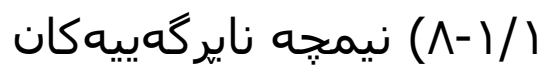

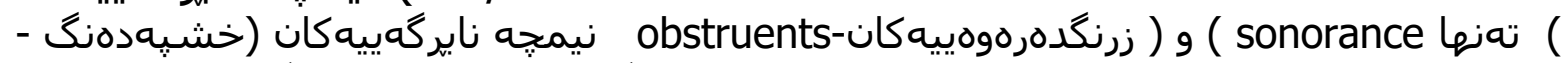

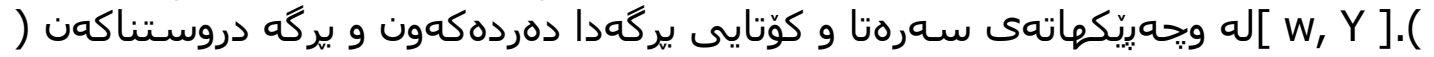

\section{(ץ/lvowels}

وهك زرنكدهرهوهيى دياريدهكرين و $\quad$ Articulation

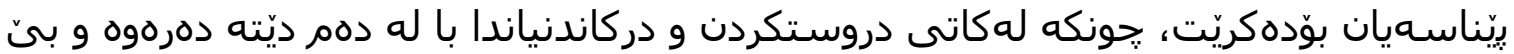

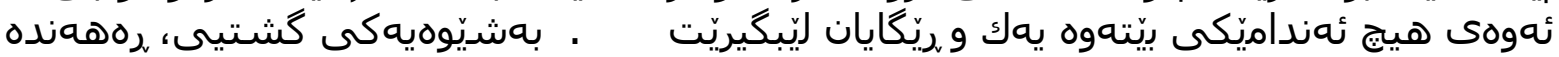

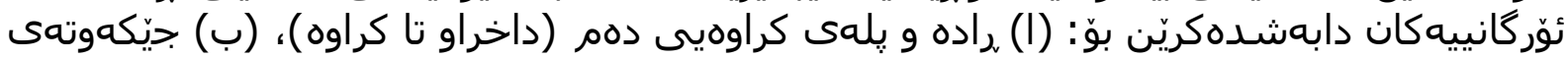

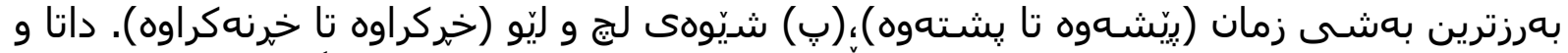

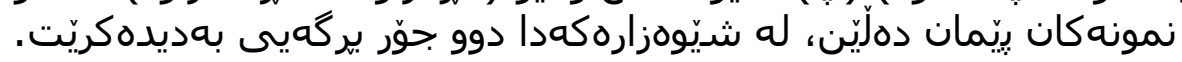

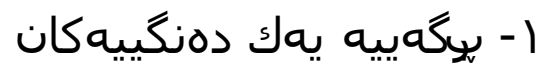

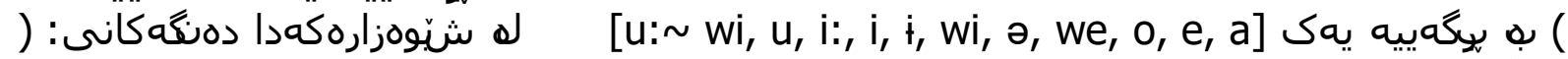

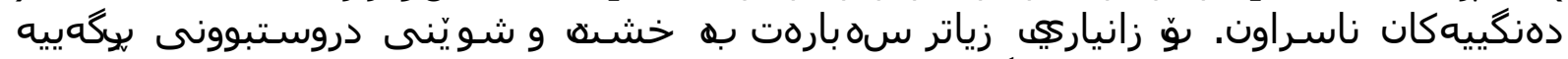
يapeter ladefoged: 2005

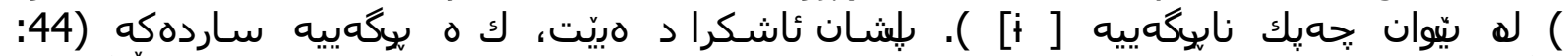

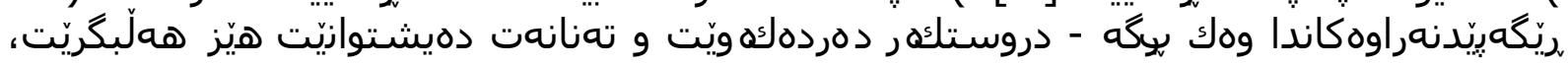
ومك:

$$
\begin{aligned}
& >\quad \text { / هاتن/ hatin< } \quad \text { [ha . t'in ] (ناوى كرداركيu) }
\end{aligned}
$$

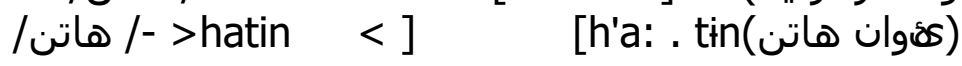

- rDiphthongs

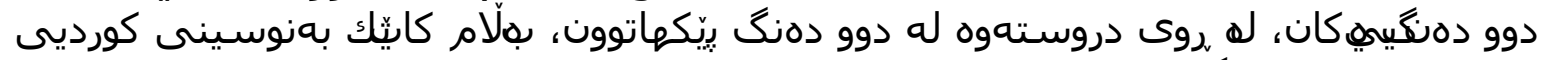

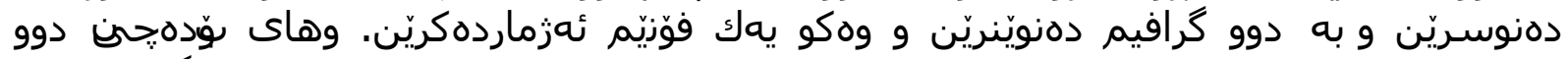

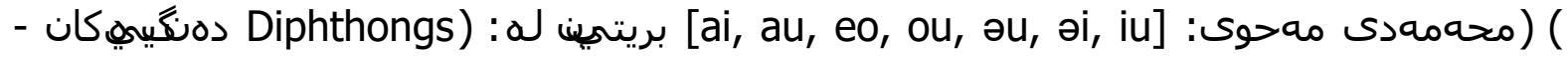
. $(\Sigma \wedge \Sigma: T \cdot 17$

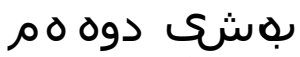

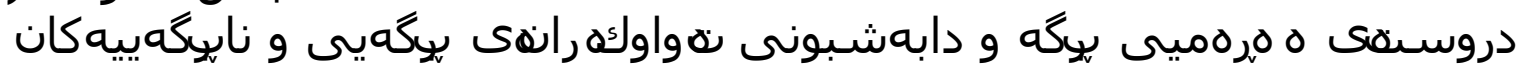

\section{(1/T) دروسته}

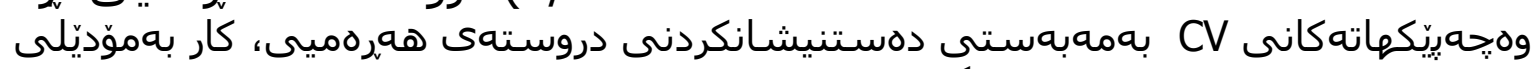

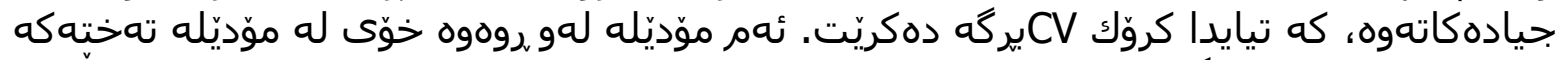

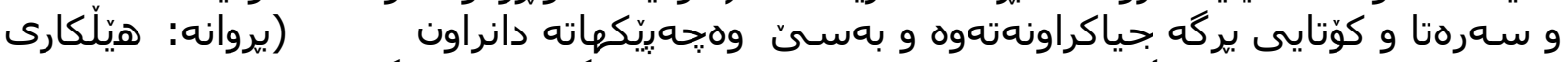

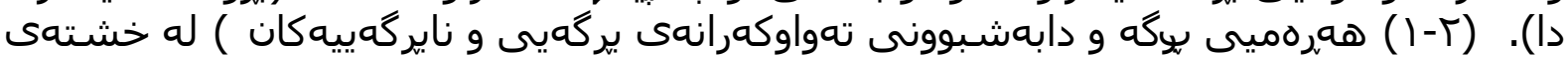

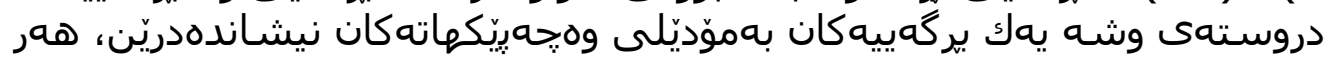

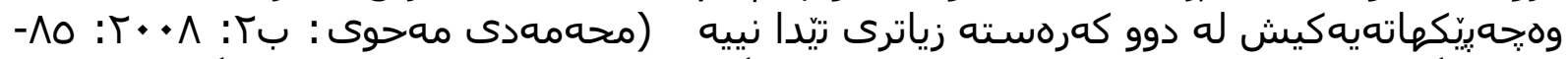

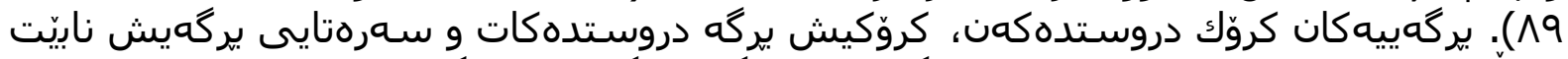

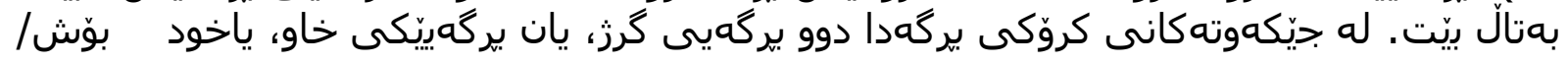




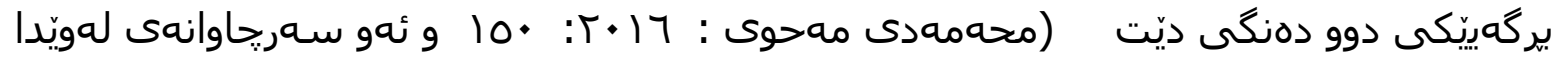

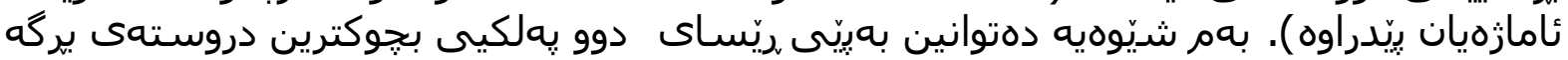

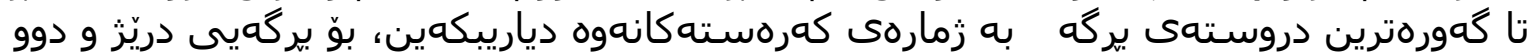

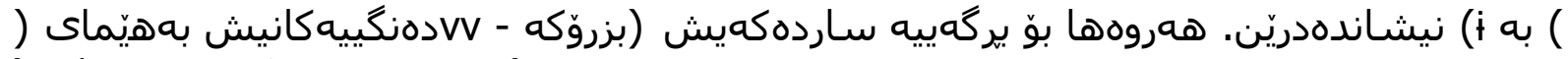

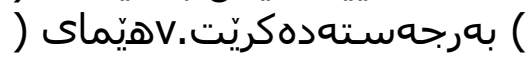

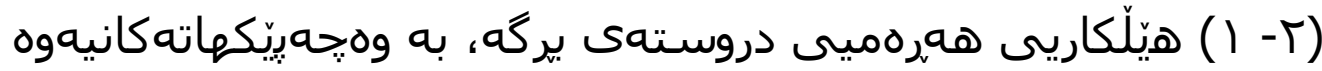

[si:]

[bri:n]

[dost]

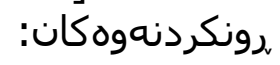

$$
\begin{aligned}
& \text { بQ } \\
& \text { سمةرةتاى بركه } \\
& \text { سمه روا بـhyme } \\
& \text { Surcleus } \\
& \text { كوّتاكي بركة }
\end{aligned}
$$

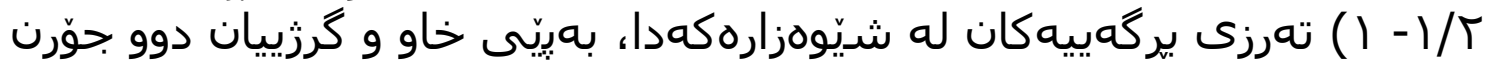

|) بركهييهكانى سـروهوه

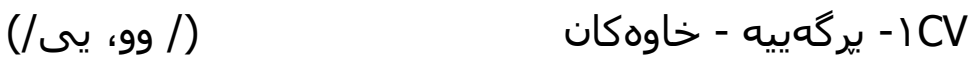

$$
\begin{aligned}
& \text { [si:], [du:], [bu: bwi] } \\
& \text { TCV }
\end{aligned}
$$

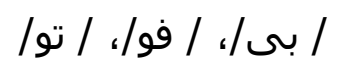

$$
\begin{aligned}
& \text { [bi], [fu pv], [tu] }
\end{aligned}
$$

ب) برگهييهكان ريزى ناوهراست و خوارهوه

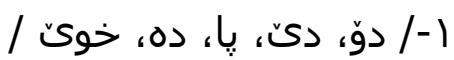

$$
\begin{aligned}
& \text { [do], [de], [pa] [pe], [də], [xœ] }
\end{aligned}
$$




$$
\begin{aligned}
& \text { r C / مار/، / تيّر/، / سهر/، / بوّر/، / دويّت /، من/ CVC } \\
& \text { [mar], [ter], [sər], [bor], [dœt],[min] }
\end{aligned}
$$

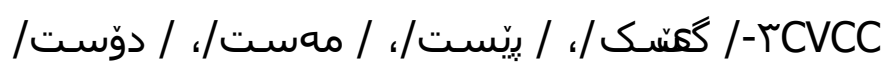

[gesk] [gisk], [pest], [məst], [dost]

ع- / كَلِيّم/، / بروّش/، / برويّن/

CCVC

[głem], [bRo]], [bzœn]

ه يَلْكاريى دهربراوى نهرزهكآ بِكه
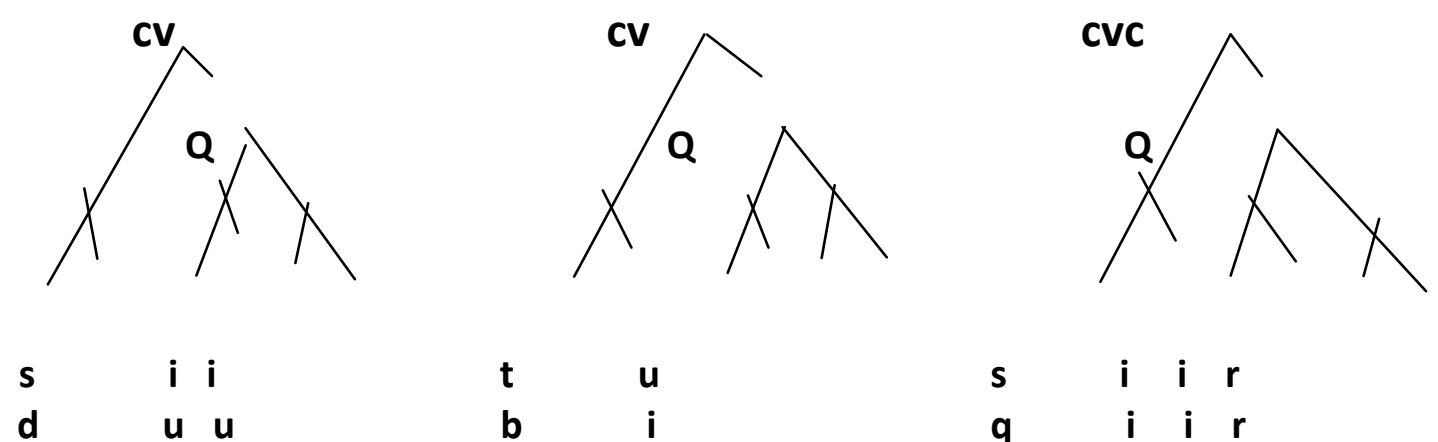

$\begin{array}{ll}\mathbf{t} & \mathbf{u} \\ \mathbf{b} & \mathbf{i}\end{array}$

s i i $r$

d u u
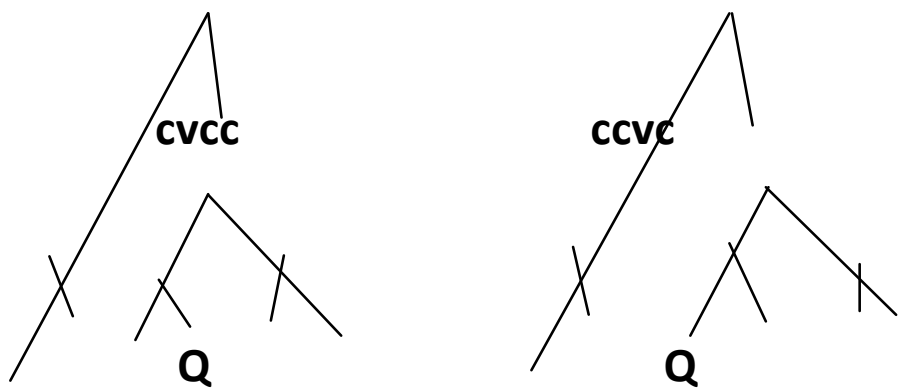

q i i r

$\begin{array}{llllllllll}\text { l. } & \mathbf{p} & \mathbf{0} & \mathbf{s} & \mathbf{t} & \mathbf{b} & \mathbf{R} & \mathbf{0} & \mathbf{0} & \mathbf{J} \\ \mathbf{a} & \mathbf{g} & \mathbf{u} & \mathbf{n} & \mathbf{d} & \mathbf{k} & \mathbf{r} & \mathbf{a} & \mathbf{a} & \mathbf{s}\end{array}$ 


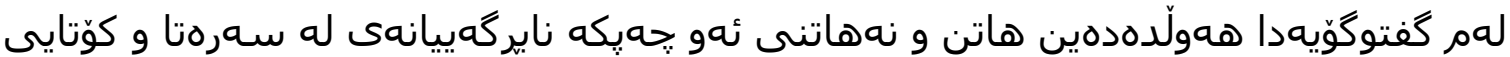

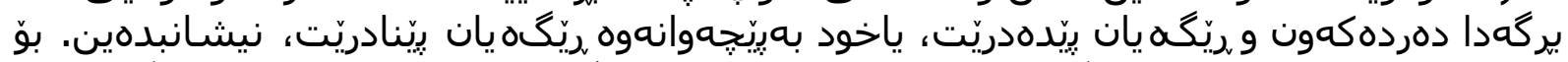

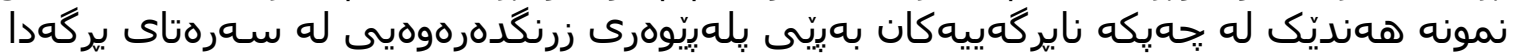

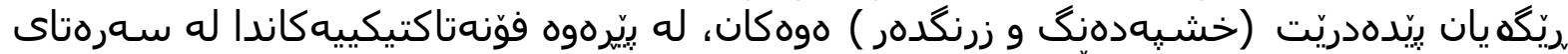

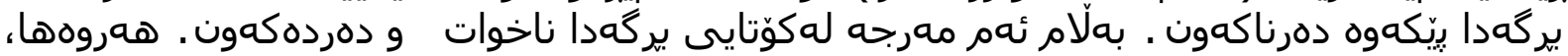

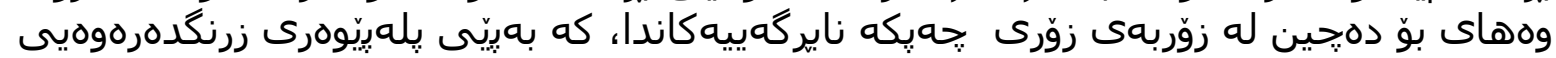

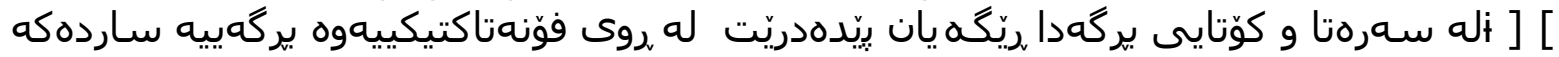

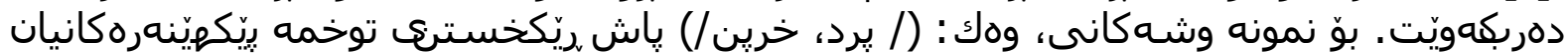

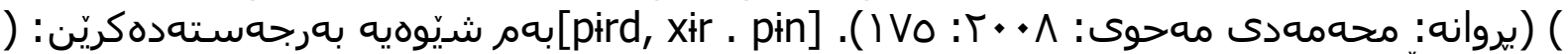

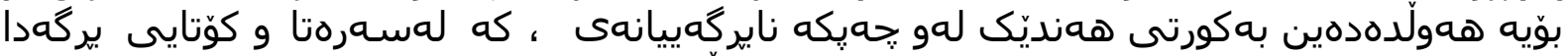

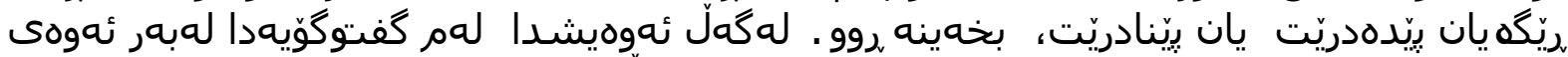

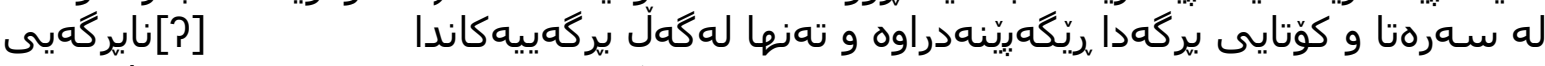
دهردهكويّيت، بوّيه نمونهى بوَ نهويّنراوهتهوه.

$$
\text { يلج بِيِوهرى زرنكدهرهوهيى }
$$

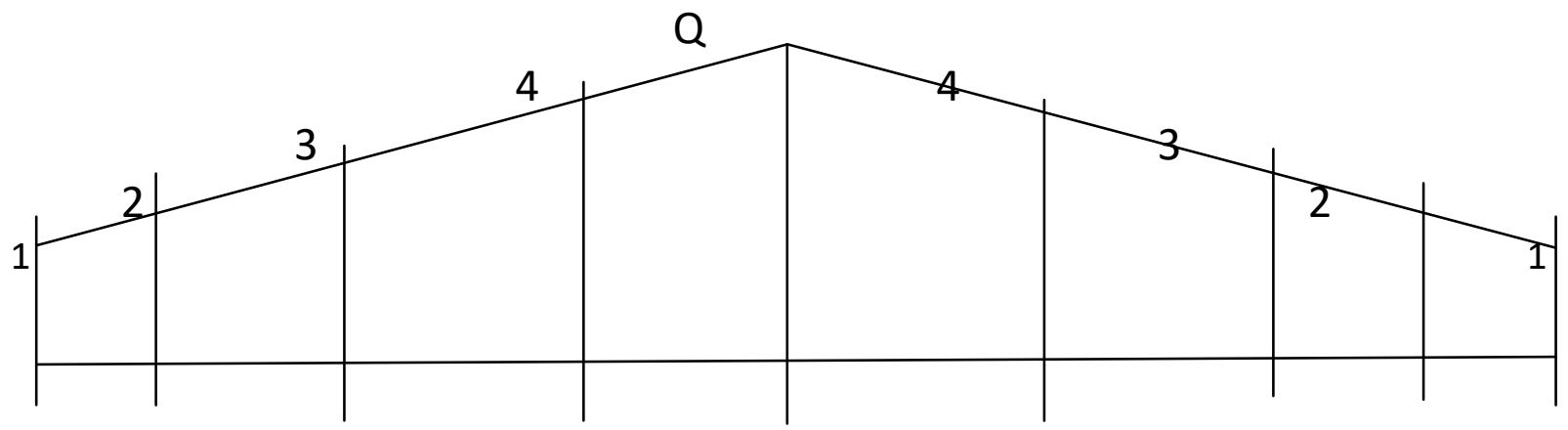

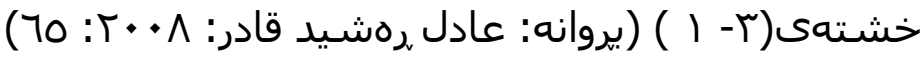

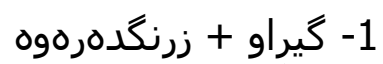

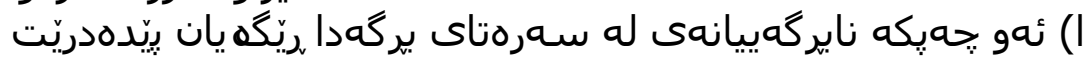

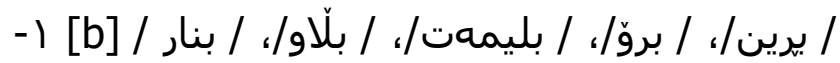

[bRi:n], [bro], [bli . mət], [błau], [bnar]

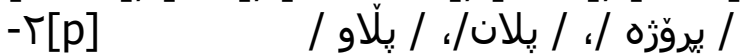

[pRo . 3ə], [pla:n], [pła:u]

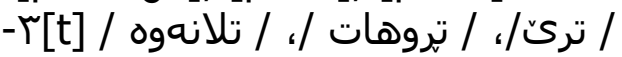

[tre], [tRu . ha:t] ,[ tla . nə . wə ]

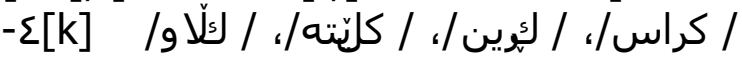

[kra:s], [kRi:n], [ kle . tə],[kła:u]

-0 [q]

[qRa:n], [qla:u]

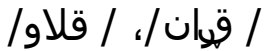

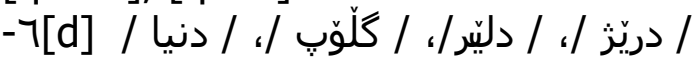

[drez], [dler], [głop], [dnya] [dunya]

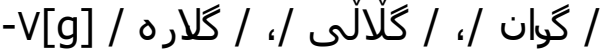

[gra:n], [gła . łi], [ gla . rə] 
ب) ئهو خهيكه نايركهييانهى له سهرةتاى يرگهدا ريّكه يان ينادريّت

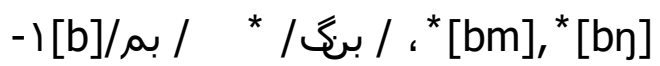

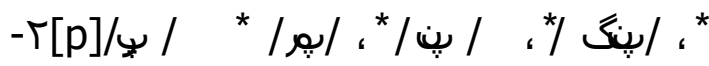

${ }^{*}[\mathrm{pr}],{ }^{*}[\mathrm{pm}],{ }^{*}[\mathrm{pn}],{ }^{*}[\mathrm{pnn}]$

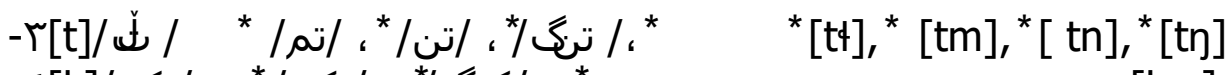

- $[\mathrm{km}],{ }^{*}[\mathrm{kn}],{ }^{*}[\mathrm{kn}]{ }^{*}$

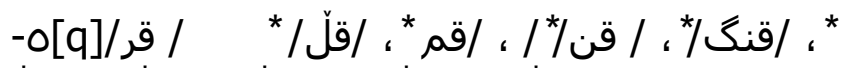

* [qr], ${ }^{*}[\mathrm{q}+],{ }^{*}$ [qm], ${ }^{*}$ [qn], * [qu]

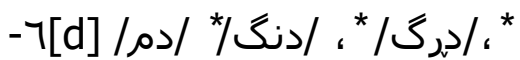

${ }^{*}[\mathrm{dm}],{ }^{*}[\mathrm{dn}],{ }^{*}[\mathrm{dRg}]$

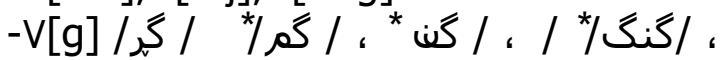

$\left.{ }^{*}[\mathrm{gR}],{ }^{*}[\mathrm{gm}],{ }^{*}[\mathrm{gn}],{ }^{*}[\mathrm{~g})\right]$

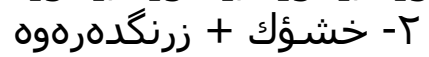

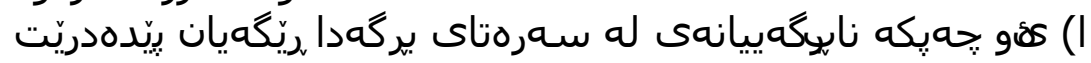

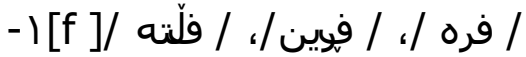

[frə], [fRi:n], [fttə]

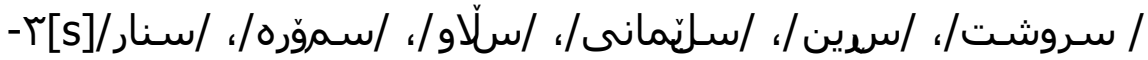

[sruft],[sRi:n], [sle . ma . ni], [słau], [smo . rə], [sna:r]

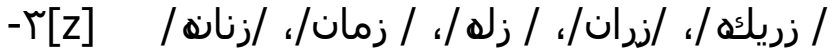

[zri: . kə], [zRa:n:], [zlə], [zma:n], [zna .nə]

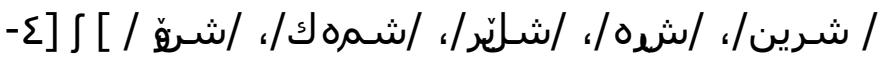

[rii:n], [Rə], []ler], [[mək], [[no]

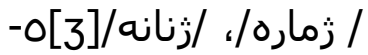

[3ma . rə], [3na .nə]

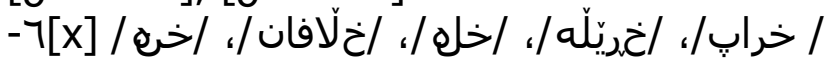

[xra:p], [xRe . \ə], [xlə], [xła . fa:n], [xnə]

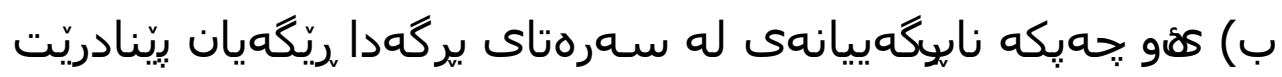

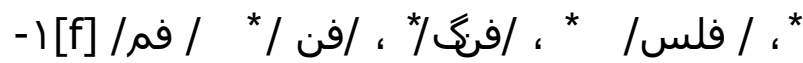

${ }^{*}[\mathrm{fm}],{ }^{*}[\mathrm{fn}],{ }^{*}[\mathrm{fn}],{ }^{*}[\mathrm{fls}]$

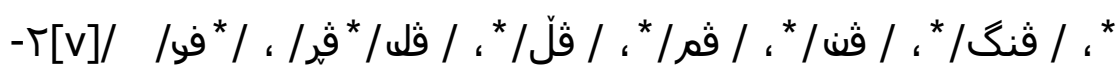

International Journal of Kurdish Studies Vol.4/1 ( January 2018) 
${ }^{*}[\mathrm{vr}],{ }^{*}[\mathrm{vR}],{ }^{*}[\mathrm{vl}],{ }^{*}[\mathrm{vq}],{ }^{*}[\mathrm{vm}],{ }^{*}[\mathrm{vn}]{ }^{*}[\mathrm{fn}]$

$-r[s] /$ / سـن

* $[\sin ]$

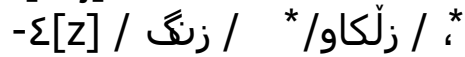

${ }^{*}[\mathrm{zr}],{ }^{*}[\mathrm{zq} \cdot \mathrm{kau}]$

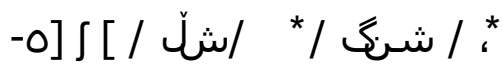

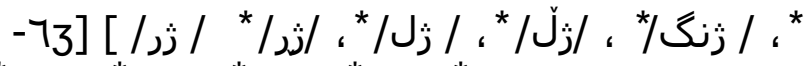

${ }^{*}[3 r],{ }^{*}[3 R],{ }^{*}[3 \mid],{ }^{*}[34],{ }^{*}[3 \mathrm{Y}]$

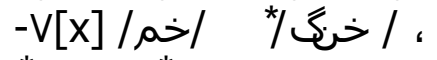

${ }^{*}[\mathrm{xm}],{ }^{*}[\mathrm{xn}]$

$-\wedge[\gamma] \quad / \quad /^{*}$

$-9[\mathrm{~h}] / \quad /^{*}$

$-1 \cdot[\mathrm{h}] /$

$-1)[\varsigma] /$

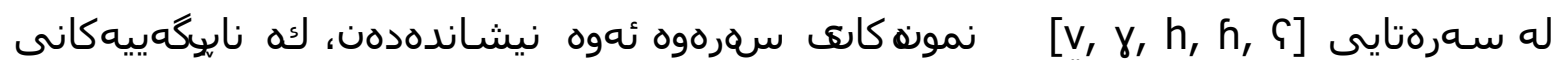

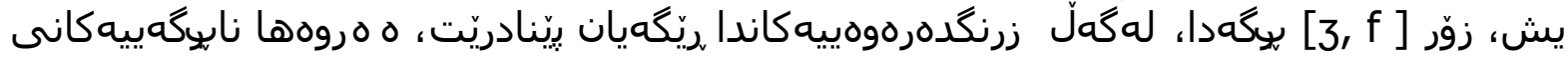
جالاك نين.

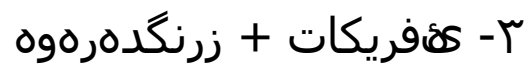

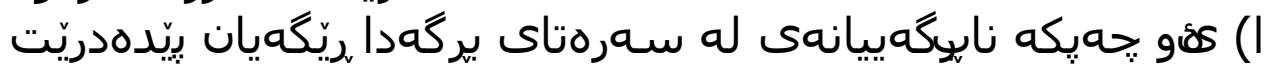

- اجرا/، /جنين/، /جله/، /جلّيّس/، إجرين/

[tra:], [t'ni:n],[tlə], [tłles], [trRi:n]

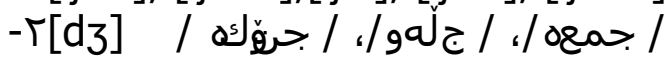

[dzmৎə dzumৎə], [dzłəu], [dzno . kə]

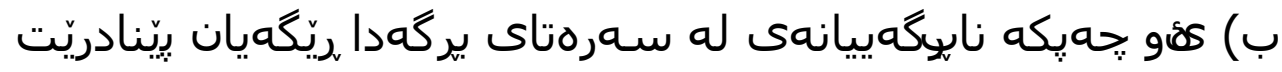

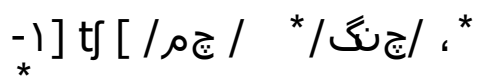

$[\mathrm{tgm}],{ }^{*}[\mathrm{tgin}]$

-

${ }^{*}[\mathrm{dg} r],{ }^{*}[\mathrm{dzR}],{ }^{*}[\mathrm{dg} 4],{ }^{*}[\mathrm{dg} n]$

ع- گيراو + خ خشوك 


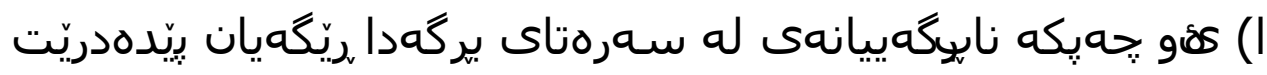

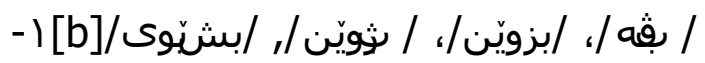

[bvə], [bzœn], [b3œn], [b]e . wi]

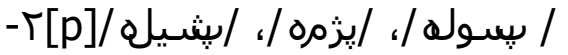

[psu . lə], [pзmə], [pлi: . lə]

$-r[\mathrm{t}]$

/ تسين/

[tsi:n]

-

[dzən]

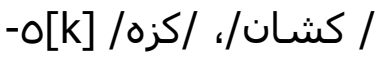

[kJa:n], [kzə]

$-7[g]$

gzik], [gvə] -V

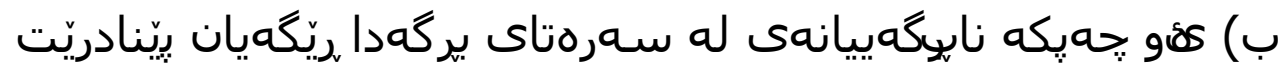

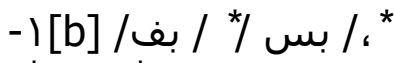

${ }^{*}[\mathrm{bf}],{ }^{*}[\mathrm{bs}]$

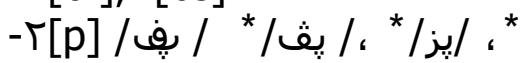

${ }^{*}[\mathrm{pf}],{ }^{*}[\mathrm{pv}],{ }^{*}[\mathrm{pz}]$

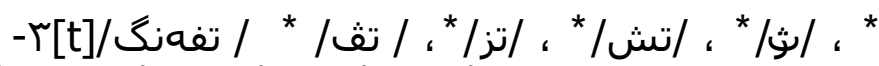

${ }^{*}[\mathrm{tf} ə \eta],{ }^{*}[\mathrm{tv}],{ }^{*}[\mathrm{t}],{ }^{*}[\mathrm{t}],{ }^{*}[\mathrm{t} 3]$

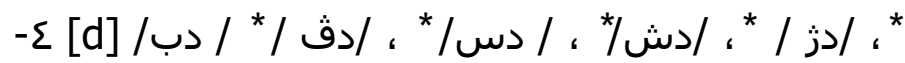

$\left.{ }^{*}[\mathrm{db}],{ }^{*}[\mathrm{dv}],{ }^{*}[\mathrm{ds}],{ }^{*}[\mathrm{~d}]\right],{ }^{*}[\mathrm{~d} 3]$

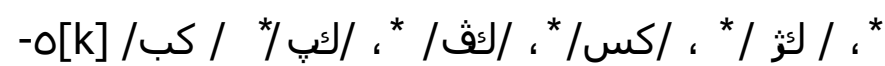

${ }^{*}[\mathrm{~kb}]{ }^{*}[\mathrm{kp}],{ }^{*}[\mathrm{kv}],{ }^{*}[\mathrm{ks}],{ }^{*}[\mathrm{k} 3[$

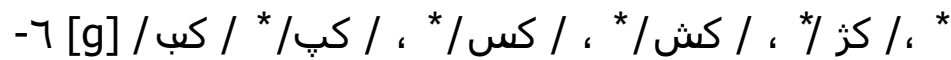

${ }^{*}[\mathrm{gb}],{ }^{*}[\mathrm{gp}],{ }^{*}[\mathrm{gs}],{ }^{*}[\mathrm{~g}] \mathrm{,}{ }^{*}[\mathrm{~g} 3]$

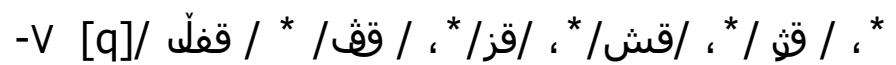

${ }^{*}$ [qft ] [qu . ft], ${ }^{*}[\mathrm{qv}],{ }^{*}[\mathrm{qz}],{ }^{*}[\mathrm{qf}],{ }^{*}[\mathrm{q} 3]$

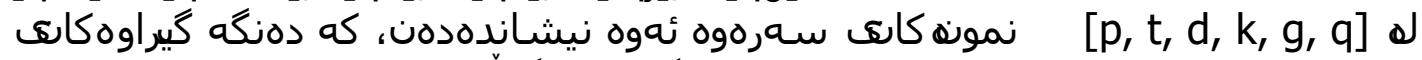

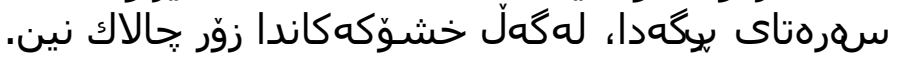

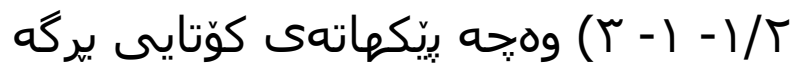

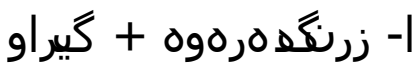




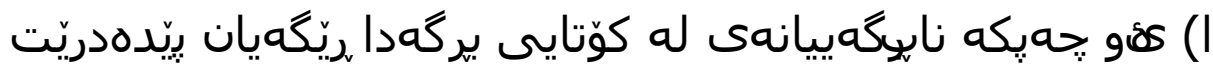

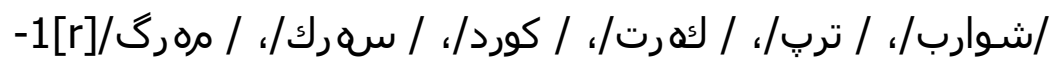

[jwarb], [tirp], [kərt], [kurd], [sərk], [məg]

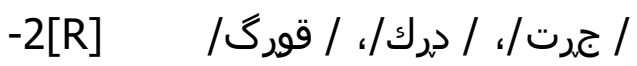

[quRi] [zoRt] [djiRt], [diRk], [qoRg]

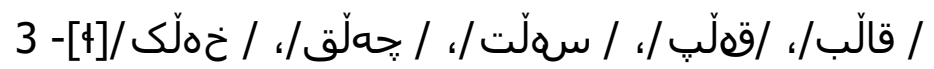

[qałb], [qəłp], [səłt], [dzəłq], [xətk]

$4-[\mathrm{m}]$

[kəmp], [məmk]

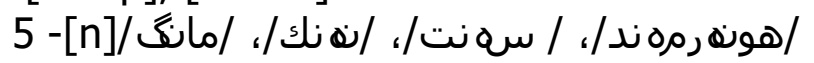

[hu , nər . mənd], [sənt], [nənk], [ma:n]

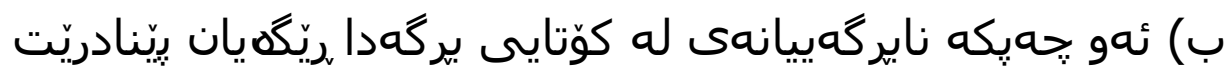

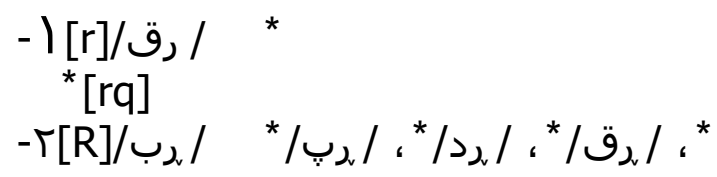

$[\mathrm{Rq}]^{*}$

$[\mathrm{Rb}],{ }^{*}[\mathrm{Rp}],{ }^{*}[\mathrm{Rd}],{ }^{*}$

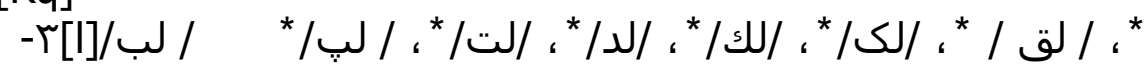

${ }^{*}[\mathrm{lb}],{ }^{*}[\mathrm{lp}],{ }^{*}[\mathrm{lt}],{ }^{*}[\mathrm{ld}],{ }^{*}[\mathrm{lq}],{ }^{*}[\mathrm{lk}],{ }^{*}[\mathrm{lg}]$

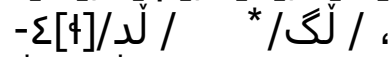

${ }^{*}[\mathrm{dd}],{ }^{*}[\mathrm{Hg}]$

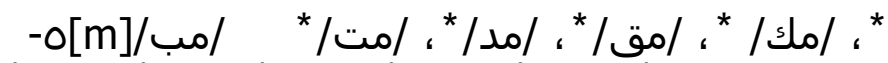

${ }^{*}[\mathrm{mb}],{ }^{*}[\mathrm{mt}],{ }^{*}[\mathrm{md}],{ }^{*}[\mathrm{mq}],{ }^{*}[\mathrm{mk}],{ }^{*}[\mathrm{mg}]$

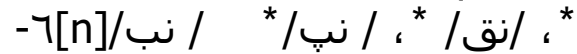

${ }^{*}[\mathrm{nb}],{ }^{*}[\mathrm{np}],{ }^{*}[\mathrm{nq}]$

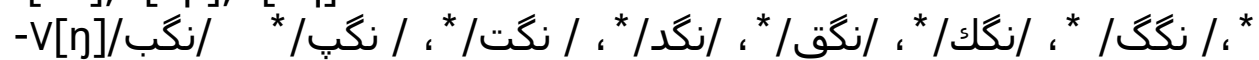

${ }^{*}[\mathrm{ngb}],{ }^{*}[\mathrm{ngp}],{ }^{*}[\mathrm{ngt}],{ }^{*}[\mathrm{ngd}],{ }^{*}[\mathrm{ngq}],{ }^{*}[\mathrm{ngk}],{ }^{*}[\mathrm{ngg}]$

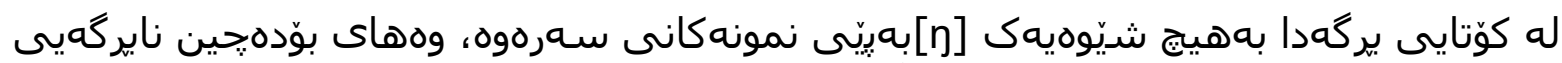

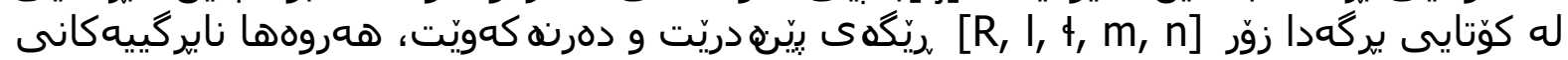
دهرنه كون.

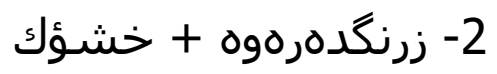

|) ئهو جهويكه نايركَييانهى له كوّتايى يرگهدا ريّكَه يان بِيدهدريَت

International Journal of Kurdish Studies Vol.4/1 ( January 2018) 


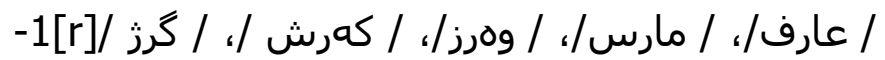

[Ca:rf], [ma:rs], [wərz], [kər]], [girz]

$-2[4]$

/ قولّف [quiff ]

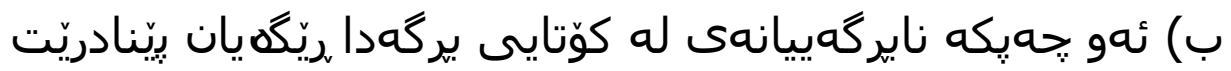

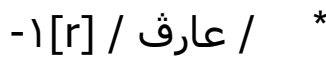

* [Ca:rv]



${ }^{*}[\mathrm{Rf}],{ }^{*}[\mathrm{Rv}],{ }^{*}[\mathrm{Rs}],{ }^{*}[\mathrm{Rz}],{ }^{*}[\mathrm{R}] \mathrm{r}^{*}[\mathrm{R} 3]$



${ }^{*}[\mathrm{If}],{ }^{*}[\mathrm{lv}],{ }^{*}[\mathrm{Is}],{ }^{*}[\mathrm{lz}],{ }^{*}[\mathrm{II}],{ }^{*}[\mathrm{I} 3]$

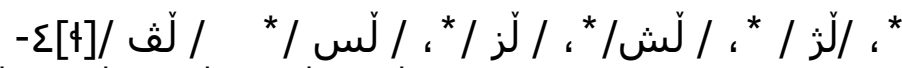

${ }^{*}[\mathrm{dv}],{ }^{*}[\mathrm{ds}],{ }^{*}[\mathrm{dz}],{ }^{*}[\mathrm{t}],{ }^{*}[\mathrm{~d} 3]$

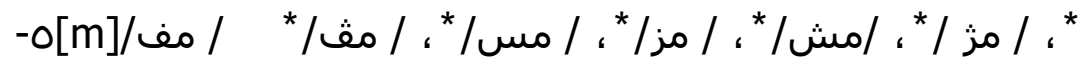

${ }^{*}[\mathrm{mf}],{ }^{*}[\mathrm{mv}],{ }^{*}[\mathrm{~ms}],{ }^{*}[\mathrm{mz}],{ }^{*}[\mathrm{~m} J],{ }^{*}[\mathrm{~m} 3]$

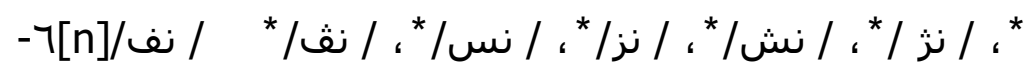

$\left.{ }^{*}[\mathrm{nf}],{ }^{*}[\mathrm{nv}],{ }^{*}[\mathrm{~ns}],{ }^{*}[\mathrm{nz}],{ }^{*}[\mathrm{n}]\right],{ }^{*}[\mathrm{n} 3]$

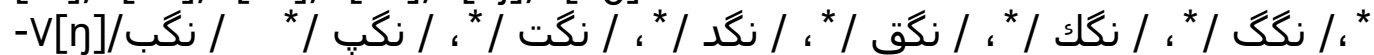

${ }^{*}[\eta \mathrm{b}],{ }^{*}[\mathrm{np}],{ }^{*}[\mathrm{nt}],{ }^{*}[\mathrm{nd}],{ }^{*}[\mathrm{nq}],{ }^{*}[\mathrm{nk}],{ }^{*}[\mathrm{gg}]$

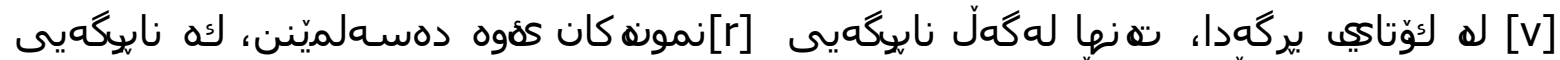

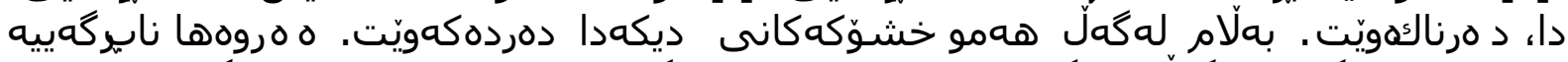

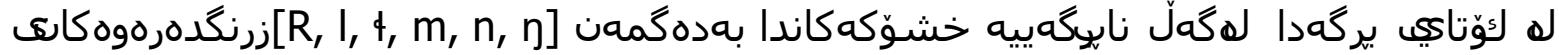
دهبينرينهوهاتو.

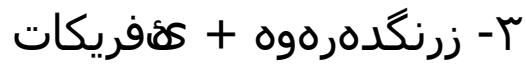

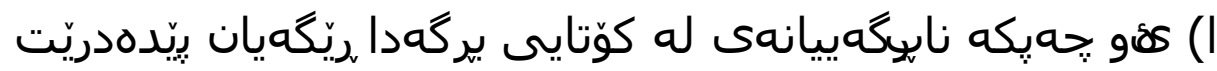

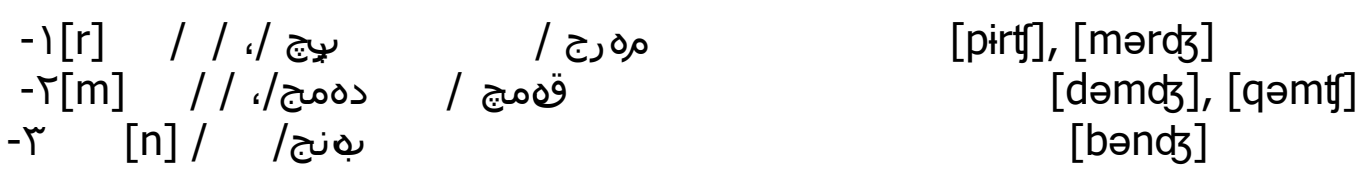

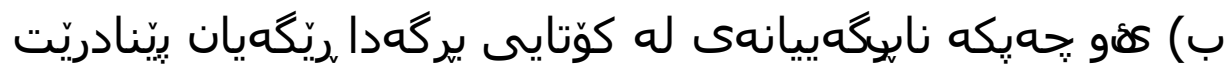




$$
\begin{aligned}
& -I[R] / \int^{2} / \text { */ब } \\
& \text { * }[\mathrm{Rt} t],{ }^{*}[\mathrm{Rd} 3]
\end{aligned}
$$

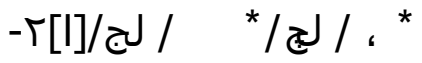

$$
\begin{aligned}
& {[\mathrm{It}],{ }^{*}[\mathrm{ld} \zeta]{ }^{*}}
\end{aligned}
$$

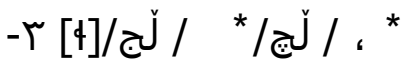

$$
\begin{aligned}
& { }^{*}[\mathrm{dt}],{ }^{*}[\mathrm{dd}] \\
& -\Sigma[\mathrm{m}] / \mathrm{O} / \text { * } \\
& {[\mathrm{mt}] \text { * }} \\
& \text {-o[n]/ بهنج/ } \\
& \text { [bəntt] * }
\end{aligned}
$$

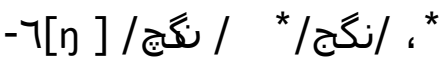

$$
\begin{aligned}
& {[n t],{ }^{*}[n d s] \text { * }}
\end{aligned}
$$

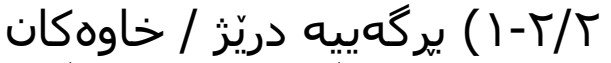

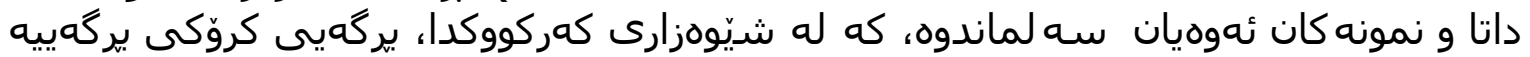

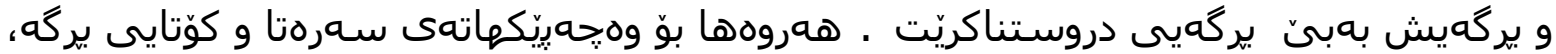

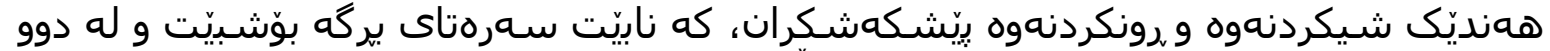

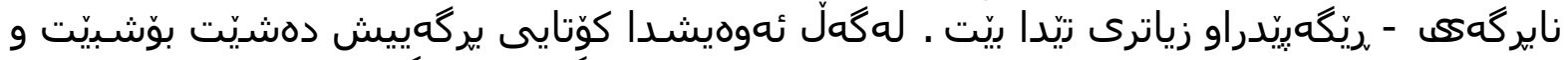

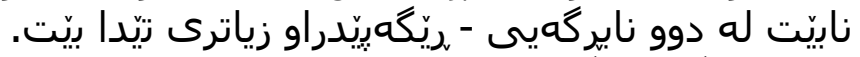

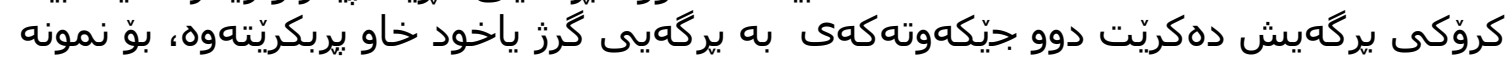

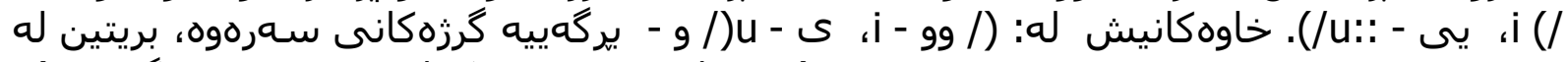

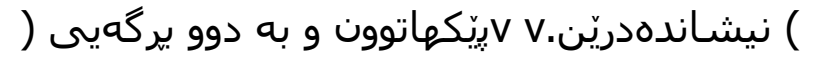

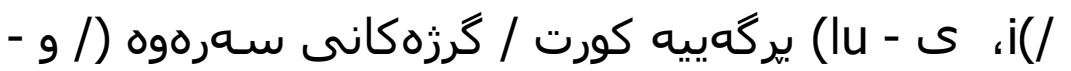

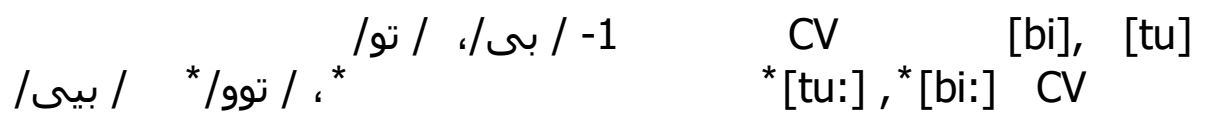

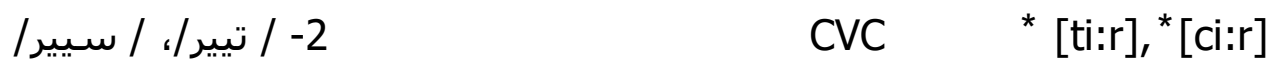

[bist], [wist], [kurd], [sruft]

$$
\text { 3CVCC }
$$

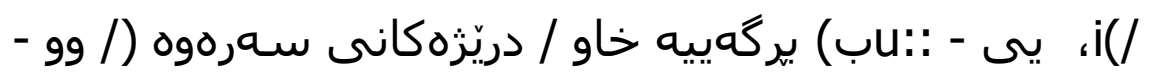

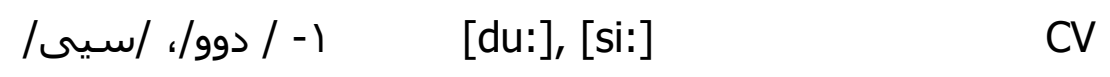

[tu:n tfwin ], [bu:n^ bwin], [si:r], [pi:r]

$$
\text { / TCVC }
$$




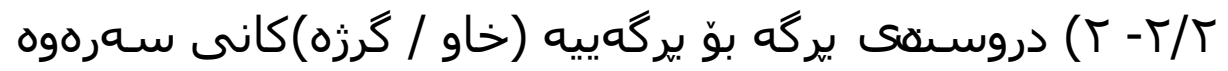
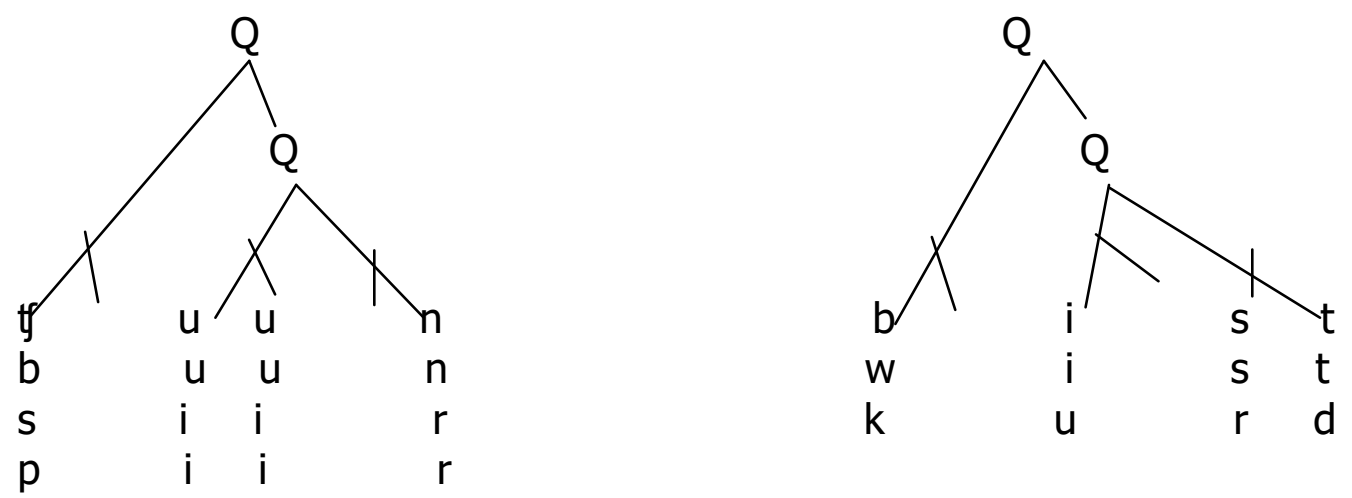

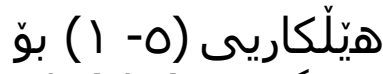

هيَلْكاريى (ع- ا ) بوّ برگهييه (كرزه)كان

برگهييه (خاو)كان (1) بون

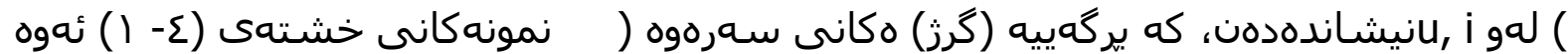

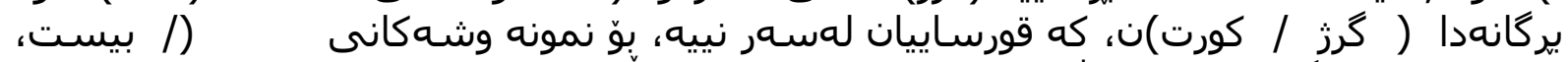

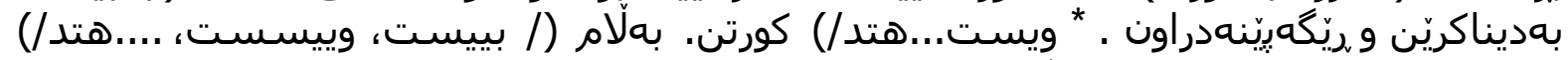

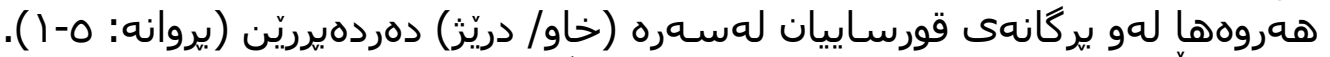

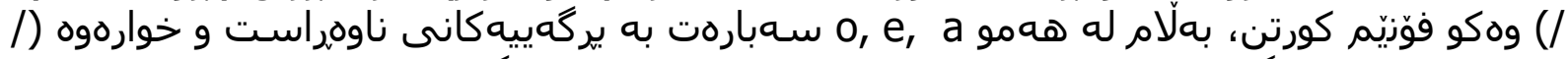

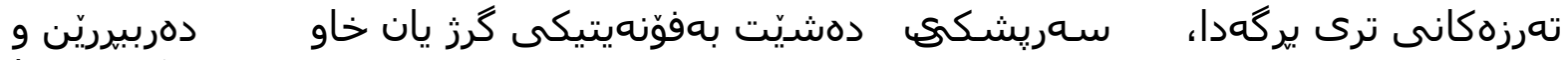
بهرجستهبكريّن، وهك

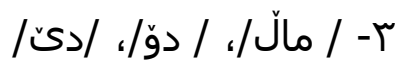

[mat] [ma:4], [do] [do:], [de] [de:]

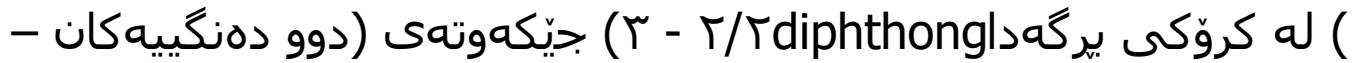

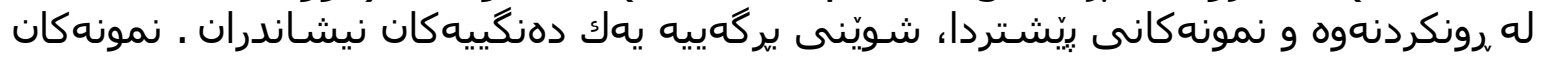

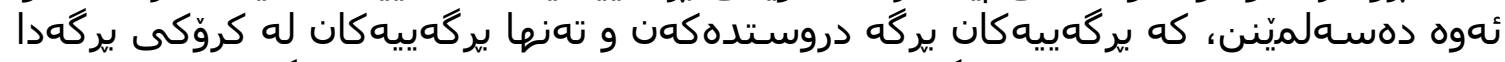

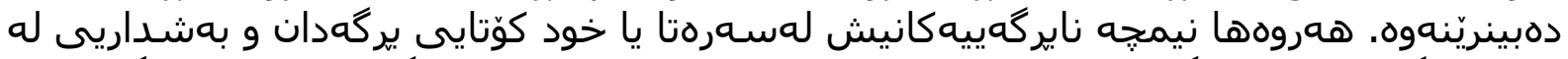

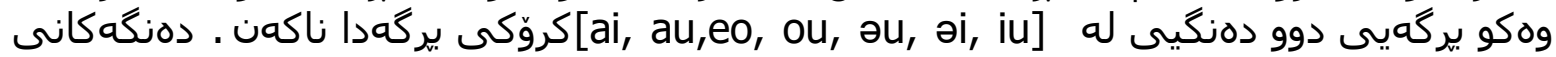

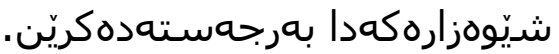

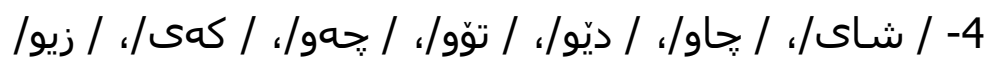

[jai],[tfau], [deu], [tou], [ţəu], [kəi], [ziu] 


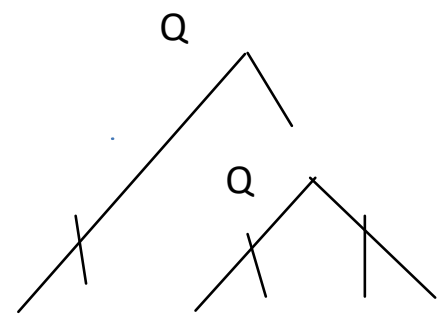

\begin{tabular}{|c|c|c|}
\hline -[Jai] & $\int$ & $a$ \\
\hline$-[\mathrm{t} a u]$ & $t$ & a \\
\hline$-[$ deu $]$ & d & $\epsilon$ \\
\hline$-[$ tou $]$ & $t$ & 0 \\
\hline$-[$ təu $]$ & t & a \\
\hline -[kəi] & k & ə \\
\hline -[ziu] & $z$ & $\mathbf{i}$ \\
\hline
\end{tabular}

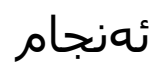

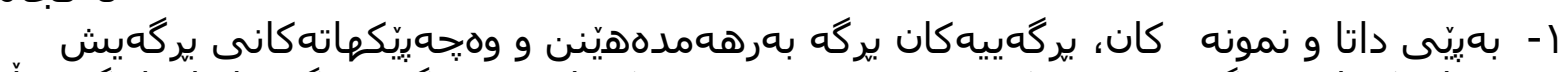

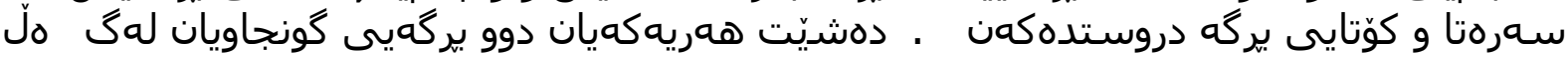

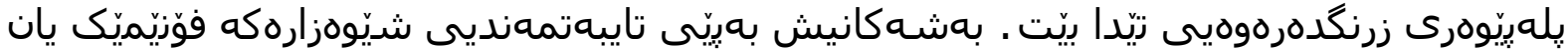

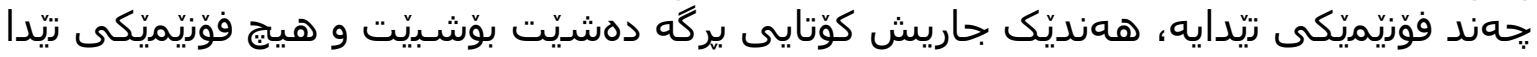

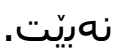

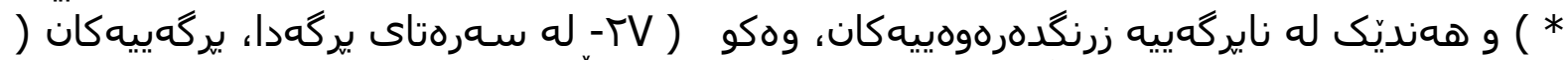

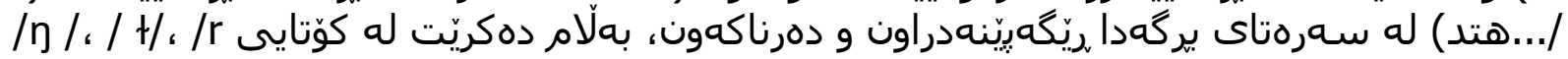

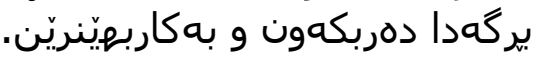

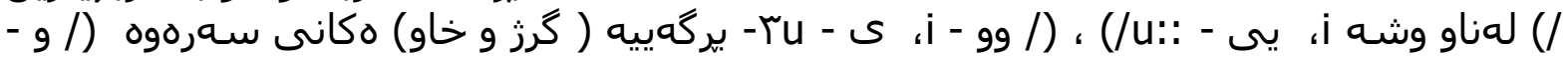

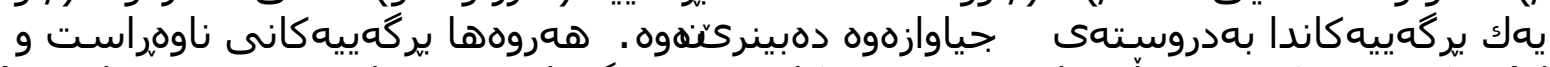

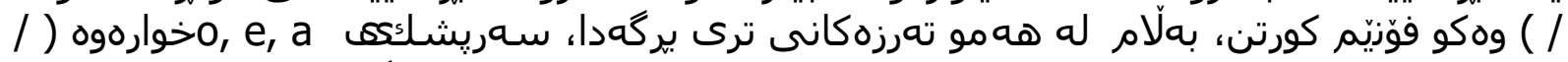

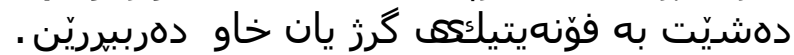

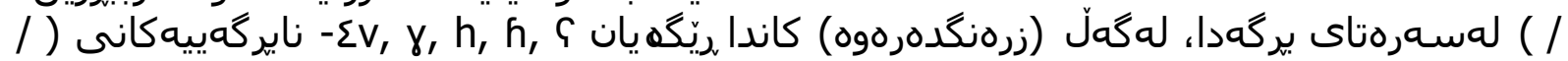

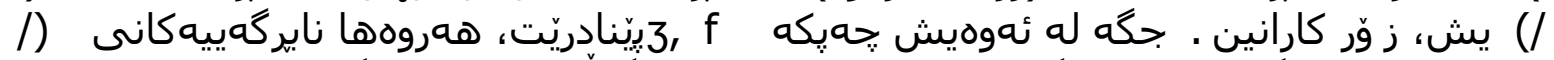

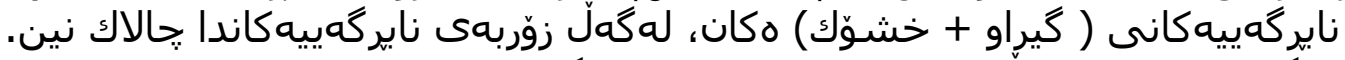

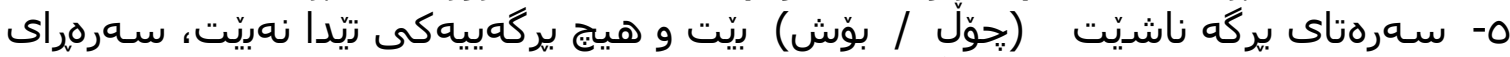

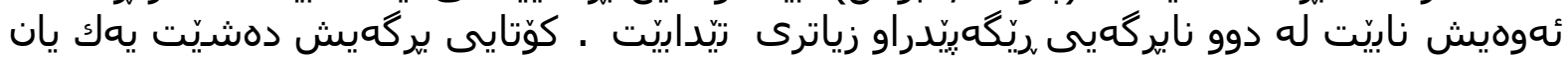

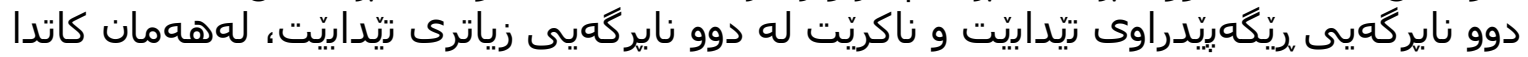

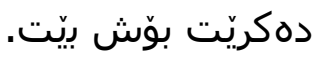

7- بهزورى جهويكه نايرگهييهكان له كوّتايى برگهدا نابينريّنهوه.

سموريجاوهكان

ا - عادل رهشـيد قادر (2008)، فوّنوّلوّرَى زمانى كوردى كرمانجى سهروو، نامهى دكتوّرا،

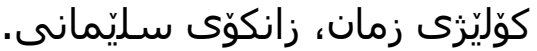

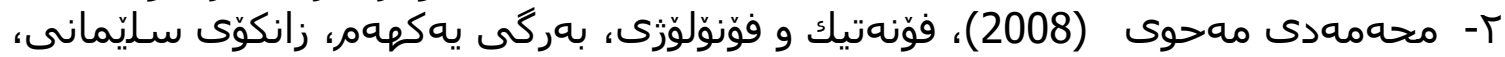
سـليّمانى.

ז- محهمهدى مهحوى (2008)، فوّنهتيك و فوّنوّلوّرَى، بهرگى دوههم، زانكوّى سليّمانى، سـليّمانى. 


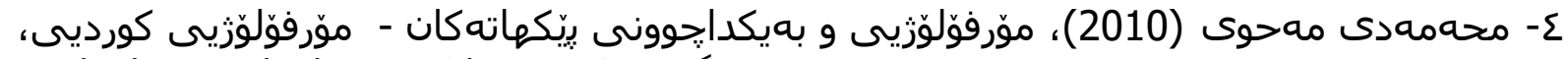

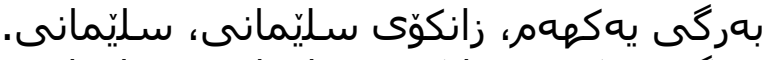

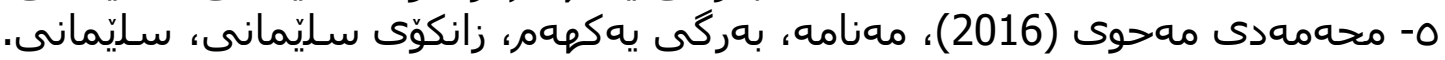

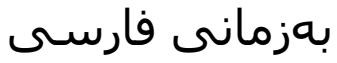

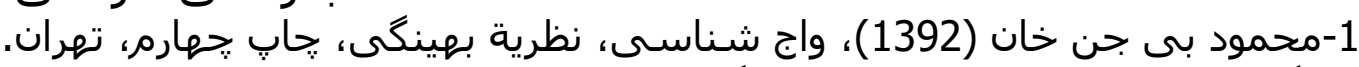

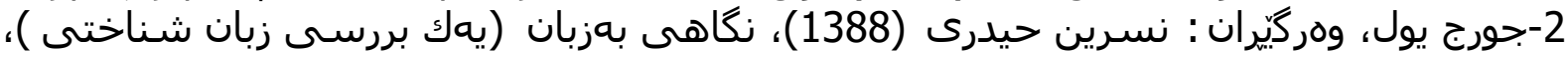
تحاب هشتهم، تهران.

بزمانى ئينكليزى 1-Ladefoged, p, (2010), (A COURSE IN PHONETICS), SIXTH EDITISON.

2-Mike Davenport and s. j. Hannahs (2005), (INTRODUCING Phonetics phonology. 